\title{
Über die Kondensationsprodukte von Phenylhydroxylamin mit Oxymethylenverbindungen und Carbinolen. IV. Methyleneampher-Phenylhydroxylamin
}

von

\author{
H. Rupe und W. Diehl.
}

(9. X. 22.)

Reduktion des Methylencampher-phenylhydroxylamines.

In der ersten Abhandlung ${ }^{1}$ ) wurde gezeigt, dass das Phenylhydroxylaminderivat sich mit Zink und Essigsäure zum Anilidomethylencampher

$$
\mathrm{C}_{8} \mathrm{H}_{14}\left\langle l_{\mathrm{CO}}^{\mathrm{C}=\mathrm{CH} \cdot \mathrm{N} \cdot \mathrm{C}_{6} \mathrm{H}_{5}}+\mathrm{H}_{2}=\mathrm{C}_{8} \mathrm{H}_{14}\left\langle\backslash_{\mathrm{CO}}^{\mathrm{C}-\mathrm{CH} \cdot \mathrm{NH} \cdot \mathrm{C}_{6} \mathrm{H}_{5}}+\mathrm{H}_{2} \mathrm{O}\right.\right.
$$

reduzieren lässt. Später wurde gefunden, dass diese Reduktion auch recht gut vermittelst Natriumbisulfit durchgeführt werden kann.

5 gr des Hydroxylaminkörpers werden in möglichst wenig Alkohol gelöst und mit $5 \mathrm{~cm}^{3}$ konzentrierter Natriumbisulfitlösung versetzt. Das Gemisch färbt sich rasch rot und nach kurzem Schütteln tritt Erwärmung ein, die sich bis zum Sieden des Alkohols steigert. Aus der schliesslich hellgelb gewordenen Lösung wird der Alkohol durch Evakuieren entfernt, dann versetzt man mit Wasser und saugt den ausgefällten Körper ab. Nach mehrmaligem Umkrystallisieren besitzt er den Smp. 165-167 des Anilidomethylencamphers; der Smp. des Gemisches mit diesem ist derselbe.

$N$-Chlorid des Methylencampher-phenylhydroxylamines.

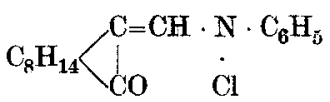

$5 \mathrm{gr}$ Hydroxylaminverbindung löst man in $50 \mathrm{~cm}^{3}$ trockenem Äther und fügt unter Kühlung mit Wasser allmählich $4 \mathrm{gr}$ Thionylchlorid (=2 Mol.) dazu. Die Lösung erwärmt sich und färbt sich braunrot.

1) Helv. 4, 388 (1921). 


\section{$-907-$}

Man lässt eine Stunde stehen, in dieser Zeit scheiden sich an den Gefässwandungen rotbraune Krystalle ab. Man versetzt mit Wasser, hebt die Ätherschicht ab und schüttelt sie vorsichtig mit Bicarbonat, dann trocknet man über Magnesiumsulfat. Das nach dem Abdestillieren des Äthers hinterbleibende Öl nimmt man in wenig Alkohol auf und versetzt mit Wasser, bis eine schwache Trübung auftritt; beim Anreiben fällt dann ein krystallinischer Körper aus. Nach mehrmaligem Umkrystallisieren aus verdünntem Alkohol schmilzt er bei 102-103 ${ }^{\circ}$. Gelblichweisse, kurze Prismen, in allen gebräuchlichen organischen Lösungsmitteln leicht löslich.

\section{0,1884 gr Subst. gaben $0,0904 \mathrm{gr} \mathrm{AgCl}$ \\ 0,1856 gr Subst. gaben 0,0932 gr $\mathrm{AgCl}$ $\mathrm{C}_{17} \mathrm{H}_{20}$ ONCl Ber. Cl 12,24\% \\ Gef. , 11,$87 ; 12,42 \%$}

Die Reaktion mit Thionylchlorid verläuft nicht einheitlich, das beweist schon die unbefriedigende Ausbeute von $50-60 \%$ der Theorie. Offenbar entsteht auch noch Methylencampherchlorid, das übrigens leicht nachgewiesen werden konnte. Das Chlor ist, wie verschiedene Versuche zeigten, sehr fest am Stickstoff gebunden.

\section{Methylencampher-p-chloranilid.}

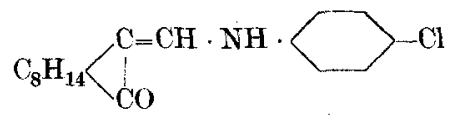

Es erschien von vorneherein durchaus nicht ganz ausgeschlossen, dass bei $\mathrm{N}$-Chlorierung des Hydroxylaminkörpers eine Umlagerung sich vollziehen kann, so nämlich, dass ein p-Chloranilid entstünde. Deshalb wurde dieser Körper von uns dargestellt, er war aber in jeder Beziehung so verschieden von dem oben beschriebenen, dass eine solche Umlagerung nicht in Betracht kommen kann.

2 gr Oxymethylencampher, gelöst in wenig Methylalkohol, wurden mit der berechneten Menge p-Chloranilin, gelöst in wenig 5-proz. Essigsäure, vermischt. Das Anilid schied sich unter Erwärmen bald nach dem Anreiben als schmutzig weisse Krystallmasse aus. Zum Umkrystallisieren verwendet man eine kalte Lösung in Aceton, welche vorsichtig mit Wasser versetzt wird. Weisse, sehr kleine Prismen vom Smp. $167-169^{\circ}$. Schwerlöslich in kaltem Ligroin.

0,1561 gr Subst. gaben 0,0791 gr $\mathrm{AgCl}$

0,3065 gr Subst gaben $0,1545 \mathrm{gr} \mathrm{AgCl}$

$\mathrm{C}_{17} \mathrm{H}_{20} \mathrm{ONCl}$ Ber. Cl 12,24\%

Gef. , 12,53; $12,47 \%$ 
Hydrobromid des Methylencampher-phenylhydroxylamines.

$$
\mathrm{C}_{8} \mathrm{H}_{14}\left\langle\begin{array}{ll}
\mathrm{CH} \cdot \mathrm{CHBr}: & \left.\mathbf{N} \cdot \mathrm{C}_{6} \mathrm{H}_{5}{ }^{1}\right) \\
\mathrm{CO} & \text { OH }
\end{array}\right.
$$

Versetzt man eine Lösung von 2,7 gr des Hydroxylaminderivates in wenig Eisessig mit einer bei $0^{0}$ gesättigten Eisessig-Bromwasserstofflösung, so beginnt das Additionsprodukt bald auszukrystallisieren, unter schwacher Erwärmung. Nach mehrstündigem Stehen wird die inzwischen festgewordene Masse abgesogen, mit Äther gewaschen und getrocknet. Gelbe Nadeln, Smp. 1210 (unter Zersetzung), schwer löslich in kaltem Benzol, Äther, Eisessig und Ligroin. Erwärmt man das Hydrobromid in Alkohol (leicht löslich), in Benzol oder Ligroin, so beginnt sogleich die Abspaltung von Bromwasserstoff; in verdünntem Alkali löst es sich klar auf, säuert man an, dann fällt ein gelber Körper aus, der nach dem Umkrystallisieren den genauen Smp. des Methylencampher-phenylhydroxylamines zeigt, also leichte Abtrennung des Bromwasserstoffes.

$$
\begin{aligned}
& 0.1564 \text { gr Subst. gaben } 0,0838 \text { gr } \mathrm{AgBr} \\
& 0,2255 \text { gr Subst. gaben } 0,1218 \text { gr } \mathrm{AgBr} \\
& 0,3008 \text { gr Subst. gaben } 10,3 \mathrm{~cm}^{3} \mathrm{~N}_{2}\left(\mathbf{1 4}^{0}, 748 \mathrm{~mm}\right) \\
& \mathrm{C}_{17} \mathrm{H}_{22} \mathrm{O}_{2} \mathrm{NBr} \text { Ber. } \mathrm{Br} 22,69 \quad \mathrm{~N} 3,97 \% \\
& \text { Gef. , } 22,80 ; 22,98,3,94 \% \\
& \text { Dibromid. } \\
& \mathrm{CBr} \cdot \mathrm{CHBr} \cdot \mathrm{N} \cdot \mathrm{C}_{6} \mathrm{H}_{5} \\
& \mathrm{C}_{8} \mathrm{H}_{14} \backslash \mathrm{CO} \quad \text { OH }
\end{aligned}
$$

Ebenso leicht wie Halogenwasserstoff wird auch Brom an das Nethylencampher-phenylhydroxylamin angelagert. Zu einer Lösung von $5 \mathrm{gr}$ der Substanz in $20 \mathrm{~cm}^{3}$ Eisessig lässt man unter Eiskühlung 2,95 gr Brom ( $=2$ Atome) in wenig Eisessig gelöst, tropfen. Die Bromfarbe verschwindet sofort und ein gelber Niederschlag wird sichtbar. Man saugt den dicken, fein krystallinischen Brei ab, wäscht mit $\ddot{A}$ ther,

1) Es wäre noch die Formel

$$
\mathrm{C}_{8} \mathrm{H}_{11}\left\langle\begin{array}{ll}
\mathrm{CBr} \cdot \mathrm{CH}_{2} \cdot \mathrm{N} \cdot \mathrm{C}_{6} \mathrm{H}_{5} \\
\mathrm{CO} & \text { OH }
\end{array}\right.
$$

möglich; wir ziehen die andere vor, da für das Additionsprodukt von $\mathrm{HBr}$ an Methyleneampher die Formel<smiles>O=CC(CBr)C(=O)c1ccccc1</smiles>
bewiesen ist. 
löst in trockenem Chloroform auf und versetzt bis zur beginnenden Trübung mit Äther. Nach einigem Stehen scheidet sich das Dibromid in feinen, hellgelben, zu Büscheln vereinigten Nadeln an den Wandungen des Kolbens ab. Smp. $117^{\circ}$ unter Zersetzung. Schwer löslich in kaltem Ligroin, Eisessig und Benzol, leicht in Äthyl- und Methylalkohol und Chloroform.

$$
\begin{array}{r}
0,1368 \text { gr Subst. gaben } 0,1186 \text { gr AgBr } \\
\mathrm{C}_{17} \mathrm{H}_{21} \mathrm{O}_{2} \mathrm{NBr}_{2} \text { Ber. Br } 37,08 \% \\
\text { Gef. , } 36,89 \%
\end{array}
$$

\section{Methylencampher-phenylhydroxylamin und Phenylhydrazin.}

$5 \mathrm{gr}$ der Verbindung werden in $15 \mathrm{~cm}^{3}$ Eisessig aufgenommen, mit einem Mol. Phenylhydrazin versetzt und darauf eine Stunde lang auf siedendem Wasserbade erhitzt. Die rote Lösung wird nach dem Erkalten auf Eis gegossen, wodurch ein rotes Öl ausfällt, das allmählich erstarrt. Beim Behandeln mit verdünnter Salzsäure geht der grösste Teil in Lösung, der ungelöste Rest (ca. 0,5 gr) zeigt nach dem Umkrystallisieren aus wenig Alkohol den Smp. $35-36^{\circ}$ sowie alle übrigen Eigenschaften des Azoxybenzoles. Aus der sauren Lösung fällt Ammoniak eine Substanz (Rohprodukt: $3 \mathrm{gr}$ ), welche nach zweimaligem Umkrystallisieren aus Aceton bei $124-125^{\circ}$ schmilzt.

$$
\begin{gathered}
0,1900 \text { gr Subst. gaben } 19,2 \mathrm{~cm}^{3} \mathrm{~N}_{2}\left(23^{\circ}, 738 \mathrm{~mm}\right) \\
\mathrm{C}_{17} \mathrm{H}_{20} \mathrm{~N}_{2} \text { Ber. } \mathrm{N} 11,11 \% \\
\text { Gef. , } 10,99 \%
\end{gathered}
$$

Analyse und die sonstigen Eigenschaften des Körpers stimmen auf das von Bishop, Claisen und Sinclair ${ }^{1}$ ) bei der Einwirkung von Phenylhydrazin auf Oxymethylencampher erhaltene Pyrazolderivat. Der Mischschmelzpunkt mit einem nach Claisen dargestellten Präparate erlitt keine Erniedrigung, ebenso gab unser Körper die Pyrazolinreaktion. Die Entstehung dieses Körpers ist so zu erklären, dass zuerst sich das Phenylhydrazon bildete, dann wurde Phenylhydroxylamin abgetrennt, das dem gewöhnlichen Schicksal der Umwandlung in Azoxybenzol anheimfiel $^{2}$ ) und der Pyrazolring gebildet:

1) A. 281,352 (1894).

2) Vergl. Helv. 4, 395 (1920). 


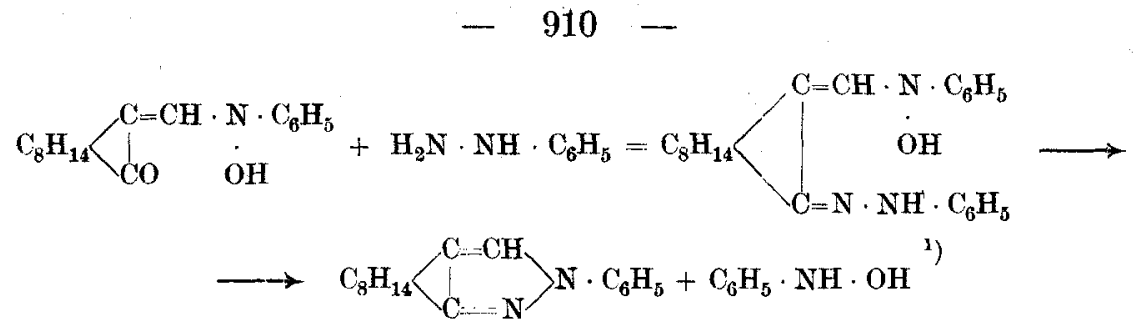

Methylencampher-phenylhydroxylamin und Semicarbazid.

$5 \mathrm{gr}$ des Hydroxylaminderivates werden, in $30 \mathrm{~cm}^{3}$ Fisessig gelöst, mit einer konzentrierten wässrigen Lösung von 3,5 gr Semicarbazidchlorhydrat und $3 \mathrm{gr}$ Kaliumacetat versetzt. Nach kurzem Stehen fällt beim Anreiben ein feinkrystallinischer Körper aus, der nach einem Tage abfiltriert wurde; aus dem Filtrate konnte mit Wasser noch mehr davon erhalten werden (Rohprodukt ca. $4 \mathrm{gr}$ ). Man löst in Eisessig unter Erwärmen, filtriert und fügt zu der erkalteten Flüssigkeit Wasser, man erhält dann das reine Produkt in Form feiner weisser Nadeln vom Smp. 205-206 .

$$
\begin{aligned}
& 0,1494 \text { gr Subst. gaben } 0,3325 \text { gr } \mathrm{CO}_{2} \text { und } 0,1080 \mathrm{H}_{2} \mathrm{O} \\
& 0,2841 \mathrm{gr} \text { Subst. gaben 0,6329 } \mathrm{gr} \text {, und 0,2022 , } \\
& 0,1740 \mathrm{gr} \text { Subst. gaben } 27,18 \mathrm{~cm}^{3} \mathrm{~N}_{2}\left(16^{0}, 745 \mathrm{~mm}\right) \\
& 0,2516 \text { gr Subst. gaben } 39,10 \mathrm{~cm}^{3} \mathrm{~N}_{2}\left(14^{0}, 740 \mathrm{~mm}\right) \\
& \mathrm{C}_{12} \mathrm{H}_{19} \mathrm{O}_{2} \mathrm{~N}_{3} \text { Ber. C 60,73 } \quad \mathrm{H} 8,07 \quad \mathrm{~N} 17,71 \% \\
& \text { Gef. , 60,72, 60,77 , 8,09, 7,96 , 17,74, 17,72\% }
\end{aligned}
$$

Der Analyse nach musste der Körper das einfache Semicarbazon des Oxymethylencamphers sein, eine Verbindung, welche schon von Wallach $^{2}$ ) dargestellt worden ist, für welche dieser Autor aber den Smp. 217-218 angibt. Wir bereiteten aus Oxymethylencampher und Semicarbazid nach seinen Angaben eine Probe des Körpers, er schmolz bei sehr langsamem Erhitzen genau bei $205-206^{\circ}$, ein MischSchmelzpunkt mit dem Semicarbazon aus Methylencampher-phenylhydroxylamin zeigte keine Erniedrigung. Zum Überflusse wurde das nach Wallach dargestellte Semicarbazon analysiert.

$$
\begin{array}{r}
0,1356 \text { gr Subst. gaben } 21,25 \mathrm{~cm}^{3} \mathrm{~N}_{2}\left(13^{0}, 724 \mathrm{~mm}\right) \\
\mathrm{C}_{12} \mathrm{H}_{19} \mathrm{O}_{2} \mathrm{~N}_{3} \text { Ber. } \mathrm{N} 17,71 \% \\
\text { Gef. , } 17,55 \%
\end{array}
$$

1) Claisen hält die Formel:<smiles>c1ccc2ncccc2c1</smiles>

für wahrscheinlich, wobei angenommen wird, dass die „Formyl“--Form des Oxymethyleneamphers reagierte; die von uns gefundene Darstellung spricht aber mehr für die oben gegebene Formel.

2) Wallach, A. 329, 129 (1903). 
Das nach dem Absaugen des Semicarbazones erhaltene Filtrat wurde alkalisch gemacht und ausgeäthert, der nach dem Verjagen des Äthers hinterbleibende, etwas schmierige, rötliche Rückstand lieferte nach dem Anreiben mit Benzin einen krystallinischen Körper, der nach weiterer Reinigung durch Umkrystallisieren sich als Phenylhydroxylamin auswies (Smp. 810, Mischschmelzpunkt usw.).

$$
\begin{aligned}
& \mathrm{C}_{8} \mathrm{H}_{11} \backslash_{\mathrm{CO}}^{\mathrm{C}=\mathrm{CH} \cdot \mathrm{N} \cdot \mathrm{C}_{6} \mathrm{H}_{5}+\mathrm{H}_{9} \mathrm{~N} \cdot \mathrm{NH} \cdot \mathrm{CO} \cdot \mathrm{NH}_{2}}=\mathrm{C}_{8} \mathrm{H}_{14} \backslash \bigcup_{\mathrm{C}=\mathrm{N}}^{\mathrm{C}=\mathrm{CH} \cdot \mathrm{OH}} \cdot \mathrm{CO} \cdot \mathrm{NH}_{2} \\
& +\mathrm{C}_{6} \mathrm{H}_{5} \cdot \mathrm{NH} \cdot \mathrm{OH}
\end{aligned}
$$

\section{Oxydation des Methylencampher-phenylhydroxylamins.}

In der früheren Abhandlung wurde schon erwähnt, dass das Hydroxylaminderivat ${ }^{1}$ ) beim Kochen mit Cupri-acetat in ein Oxydationsprodukt übergeführt wird. Die Reaktion mit Kupfersalz erfolgt übrigens nicht glatt, sondern liefert viel schmierıge Produkte; weit besser verläuft die Oxydation mit Kaliumferricyanid in alkalischer Lösung.

2,7 gr des Phenylhydroxylaminderivates werden in einer Reibschale mit wenig Alkohol angerieben und sodann mit überschüssiger verdünnter Natronlauge behandelt, es erfolgt sofort Lösung, da diese aber bei älteren Präparaten häufig nicht ganz klar ist, so wird rasch filtriert und dás Filtrat mit Eis gut gekühlt. Langsam unter Umrühren fügt man $\mathrm{zu}$ ihm eine eiskalte wässrige Lösung von 3,4 gr Kaliumferricyanid, sogleich bildet sich ein fleischroter Niederschlag; wenn in einer filtrierten Probe auf weiteren Zusatz des Oxydationsmittels kein Niederschlag mehr entsteht, wird der dicke Brei abgesogen und mit Wasser gründlich ausgewaschen. Die Ausbeute an getrocknetem, allerdings noch sehr unreinem Rohprodukt beträgt ca. 2,5 gr.

Zur Reinigung löst man die Substanz in kaltem Benzol, filtriert und überlässt die Lösung der langsamen Verdunstung; nach mehrmaliger Wiederholung dieses Verfahrens erhält man grosse, wohl aus-

1) Es sei bei dieser Gelegenheit auf ein interessantes Gegenstück zum Methylencampher-phenylhydroxylamin hingewiesen, wir meinen damit das von Bamberger und Tschirner und später von Ley zuerst aus Formanilid und Phenylhydroxylamin dargestellte:

$$
\mathrm{C}_{6} \mathrm{H}_{5} \cdot \mathrm{N}=\mathrm{CH} \cdot \mathrm{N} \cdot \mathrm{C}_{6} \mathrm{H}_{5}
$$

$\mathrm{OH}$

(Diphenyl-N-oxyformamidin). Dieser Körper gibt ein Kupfersalz, das man umkrystallisieren kann; beim Kochen mit Ferrichlorid entsteht Nitrosobenzol. Die Substanz, welche die Eigenschaften einer Base hat, wird von Natronlauge in der. Kälte nicht. angegriffen (B. 35, 714, 1451 (1902). 
gebildete, farblose, monokline Prismen, welche unter Zersetzung bei $206-208^{\circ}$ schmelzen, wobei bemerkt sei, dass der Smp. sehr von der Geschwindigkeit des Erhitzeus abhängt.

$$
\begin{aligned}
& 0,2140 \text { gr Subst. gaben } 0,5900 \text { gr } \mathrm{CO}_{2} \text { und } 0,1487 \text { gr } \mathrm{H}_{2} \mathrm{O} \\
& 0,2649 \mathrm{gr} \text {. Subst. gaben } 0,7327 \mathrm{gr} \mathrm{CO}_{2} \text { und } 0,1798 \mathrm{gr} \mathrm{H}_{2} \mathrm{O} \\
& 0,1960 \text { gr Subst. gaben } 0,5431 \text { gr } \mathrm{CO}_{2} \text { und } 0,1279 \text { gr } \mathrm{H}_{2} \mathrm{O} \\
& 0,2057 \mathrm{gx} \text { Subst. gaben } 9,3 \mathrm{~cm}^{3} \mathrm{~N}_{2}\left(18^{0}, 742 \mathrm{~mm}\right) \\
& 0,2155 \text { gr Subst. gaben } 9,7 \mathrm{~cm}^{3} \mathrm{~N}_{2}\left(18^{\circ}, 738 \mathrm{~mm}\right) \\
& \mathrm{C}_{34} \mathrm{H}_{40} \mathrm{O}_{4} \mathrm{~N}_{2} \text { Ber. C } 75,51 \quad \text { H } 7,45 \quad \text { N } 5,18 \% \\
& \text { Gef. ,, 75,21; 75,46; 75,59 ,7,77; 7,59; 7,30 , 5,07; 5,02\% }
\end{aligned}
$$

\section{Bestimmung des Molekulargewichtes.}

I. 0,2742 gr Subst. gaben in $11,48 \mathrm{em}^{3}$ Benzol bei $15,3^{\circ}$ (sp. Gew. des $\mathrm{C}_{6} \mathrm{H}_{6}$ bei $15,3^{0}=0,88672$ ) eine Gefrierpunktserniedrigung von $0,245^{\circ}$.

II. 0,2080 gr Subst. gaben in $11,11 \mathrm{~cm}^{3}$ Benzol bei $14,5^{0}$ (sp. Gew. des $\mathrm{C}_{6} \mathrm{H}_{6}$ bei $14,5^{0}=0,88800$ ) eine Gefrierpunktserniedrigung von $0,188^{\circ}$.

$$
\mathrm{C}_{34} \mathrm{H}_{40} \mathrm{O}_{4} \mathrm{~N}_{2} \text { Mol.-Gew. Ber. } 540 \text { Gef. 549,7; 560,7. }
$$

Der neue Körper ist leicht löslich in Benzol, Toluol, Chloroform, Tetrachlorkohlenstoff und Schwefelkohlenstoff, ziemlich schwer in kaltem Äther und Essigester, schwer löslich in Äthyl- und Methylalkohol, sehr schwer in Benzin, unlöslich in Alkalien.

Nach verschiedenen anderen Methoden lässt sich dieses Oxydationsprodukt noch erhalten, allerdings sind das alles Reaktionen, welche sich nicht zur Darstellung eignen, weil bei ihnen stets nur relativ kleine Mengen entstehen.

Lässt man z. B. das Phenylhydroxylaminderivat einige Wochen in Eisessiglösung stehen, so scheidet sich das Oxydationsprodukt allrnählich in grossen Prismen ab (Zersetzungspunkt 205-206 ${ }^{\circ}$ ).

Löst man das Phenylhydroxylaminderivat in verdünntem Alkali auf, so trübt sich diese Lösung bald beim Stehen an der Luft, wobei der Geruch nach Nitrobenzol sich deutlich bemerkbar macht. Nach und nach scheiden sich Flocken ab, welche nach dem Umkrystallisieren aus Benzin den Smp. 205-2060 (Zersetzung) zeigen.

Schon in der früheren Abhandlung wurde erwähnt, dass man das Oxydationsprodukt auch erhält, wenn man das Phenylhydroxylaminderivat versucht vermittelst Essigsäure-anhydrid zu acetylieren. Löst man nämlich jenen Körper in Essigsäure-anhydrid und erwärmt auf dem Wasserbade, so tritt plötzlich eine heftige Reaktion ein, die gelbe Farbe schlägt in Braunrot um und aus der heissen Lösung krystallisieren in wenigen Sekunden schöne Nadeln oder Prismen aus, die aus 


\section{- 913}

dem Oxydationsprodukt bestehen (Ausbeute ca. 30\% d. Th.). Daneben konnte nur noch etwas Acetylderivat und Harz aufgefunden werden.

Krystallographisches vom Methylencampher-phenylhydroxylamin.

Der Körper krystallisiert in kurzsäuligen-holoedrischen Krystallen.

Flächenausbildung.

1. $0=$ Basis $(001)$ OP

2. $\mathbf{a}=$ Prisma $(10 \overline{1} 0) \infty \mathrm{P}$

Flächenwinkel.

$0: a=(001):(10 \overline{10})=90^{\circ}$

$a: a=(10 \overline{10}):(10 \overrightarrow{10})=120^{\circ}$

Krystallographische Konstanten. Nicht bestimmbar, weil keine allgemeine Formen (dihexagonale Pyramiden) ausgebildet.

Optisches: Optischer Charakter: negativ. Doppelbrechung: sehr hoch.

\section{Krystallographisches vom Oxydationsprodukte ${ }^{1}$ ).}

Der zur Untersuchung gelangte Körper krystallisiert in Form von monoklin-holoedrischen Krystallen, säulig in der Richtung der b-Achse.

Folgende Flächen treten auf:

1. Pinakoide:

$\mathrm{c}=(001)$ Basis OP

$a=(100)$ Makropinakoid $\infty P \bar{\infty}$

2. Domen und Prismen:

$\dot{r}=$ (101) Makrodoma $P \bar{\infty}$

$\mathrm{p}=(\mathbf{1 3 0})$ abgeleitetes Prisma $\propto \mathrm{P} \dot{3}$

Flächenwinkel: $\quad \mathrm{c}: \mathrm{a}=(001):(100)=103^{\circ} 50^{\prime}$

$r: a=(101):(100)=130^{\circ} 30^{\prime}$

$\mathrm{c}: \mathrm{r}=(001):(101)=125^{\circ} 40^{\prime}$

$a^{\prime}: p^{\prime}=(001):(130)=107^{\circ} 50^{\prime}$

$p: p^{\prime}=(130):(130)=141^{0} 18^{\prime}$

$p: a=(130):(100)=110^{\circ} 50^{\prime}$

$c^{\prime}: q^{\prime}=(001):(132)=118^{0} 10^{\prime}$

$q^{\prime}: p^{\prime}=(132):(130)=147^{\circ} 42^{\prime}$

$\mathrm{p}^{\prime}: \mathrm{c}=(130):(001)=94^{0} 11^{\prime}$

$\mathrm{c}: \mathrm{q}=(001):(132)=118^{\circ} 18^{\prime}$

$q^{\prime}: p^{\prime}=(132):(130)=147^{0} 38^{\prime}$

$p^{\prime}: c^{\prime}=(130):(001)=94^{0} 15^{\prime}$

Krystallographische Konstanten:

1. $\beta=103^{\circ} 50^{\prime}$ (a:c)

2. Achsenverhältnis: $a$ : $b$ : 0 0,9971: $1: 1,065$

Spaltbarkeit: Nach (100) ausgezeichnet; nach (101) erkennbar.

1) Die krystallographischen Untersuchungen dieses Körpers, wie auch die des Methylencampher-phenylhydroxylamins. wurden in verdankenswerter Weise von den HH. Prof. Dr. H. Preiswerk und cand. phil. M. Grütter im mineralogischen Institut der Universität Basel durchgeführt. 
Optisches. Lage der Achse: (010); optischer Charakter: negativ; Austritt der ersten Vittellinie: fast senkrecht auf (100) $2 \mathrm{~V}=6^{\circ}{ }^{\circ} 30^{\prime}$ (mittlerer Brechungsexponent). $=1,598$.

Um einen Einblick in die Reaktion, welche sich hier vollzog, zu gewinnen, musste vor allem festgestellt werden, wie viel Sauerstoff auf eine Molekel des Hydroxylaminderivates verbraucht wurde. Das liess sich auf folgendem Wege erreichen: Zur Oxydation wurde eine bestimmte Menge (ein kleiner Üperschuss) Kaliumferricyanid angewendet, dann wurde vermittelst Kaliumpermanganat-Titration festgestellt, wie viel Kaliumferrocyanid entstanden war.

4,22 gr Kaliumferrocyanid, im Liter gelöst, bildeten eine 0.1-n. Lösung, auf diese wurde die 0,1-n. Lösung des Permanganates eingestellt, $10 \mathrm{~cm}^{3}$ der ersteren Lösung verbrauchten $10,15 \mathrm{~cm}^{3}$ Permanganat ${ }^{1}$ ). 2,7 gr Methylencampher-phenylhydroxylamin wurden in alkalischer Lösung mit 3,4 gr Kaliumferricyanid oxydiert. Der Niederschlag wurde nach dem Absaugen vollkommen ausgewaschen, das Filtrat erwärmt zur Entfernung des Alkohols, dann auf $500 \mathrm{~cm}^{3}$ aufgefüllt, es stellte also eine 0,02-n. Lösung vor. Je $50 \mathrm{~cm}^{3}$ davon wurden mit der eingestellten Permanganatlösung titriert, verbraucht (im Mittel mehrerer Bestimmungen) $10,52 \mathrm{~cm}^{3}$, ber. $10,15 \mathrm{~cm}^{3}$.

Mehrere Versuchsreihen stimmten gut überein, so dass zweifellos aus ihnen hervorgeht, dass auf 2 Molekel des Hydroxylaminderivates gerade 1 Atom Sauerstoff verbraucht wird.

Dazu kommt noch das Ergebnis der Mol.-Gew.-Bestimmuny, welche deutlich zeigt, dass der neue Körper aus zwei Molekeln des alten entstanden ist.

Tetrabromid des Oxydationsproduktes. Zu einer Lösung von $2 \mathrm{gr}$ ganz reinem Oxydationsprodukt in trockenem Chloroform fügte man in einer Kohlendioxydatmosphäre und unter peinlichstem Abschluss der Luftfeuchtigkeit 4 Atome $=1,18 \mathrm{gr}$ Brom, wobei mit Eis gekühlt wurde. Anfangs verschwand die Bromfarbe rasch, allmählich aber färbte sich die Flüssigkeit tief rot, obgleich mit Kaliumjodidstärkepapier keine Spur von freiem Brom nachgewiesen werden konnte, und dies war auch der Fall, als nach etwa einer Stunde alles Brom eingetragen war. Ebenso war Bromwasserstoff nur in Spuren aufzufinden. Zweifellos sind also 4 Atome Brom angelagert worden. Die Chloroformlösung wurde nun in einer Kohlendioxydatmosphäre mit Äther vermischt, worauf das Tetrabromid als orangerotes Pulver ausfiel. Unter Vermeidung von Luft- und Feuchtigkeitszutritt wurde rasch

1) Der bei der Oxydation des Ferro- zum Ferrisalz eintretende Farben-Umschlag von hell-grüngelb zu hell-lachsrot lässt sich gut, auch bei künstlichem Lichte, beobachten, Fehler höchstens $0,1-0,2 \mathrm{~cm}^{3}$. 
durch einen Gooch-tiegel abgesogen und im Exsikkator über Ätzkalk und Paraffinschnitzeln getrocknet. Das Tetrabromid ist leicht löslich in Methyl-, Äthylalkohol; Aceton, Chloroform; schwer löslich in Äther und Benzin. An eine vollständige Reindarstellung war angesichts der Zersetzlichkeit des Körpers in jeglichen Lösungsmitteln nicht zu denken. Der Schmelzpunkt (verwendet wurde das Analysenpräparat) des Tetrabromides ist sehr unscharf. Zwischen 65 und $75^{\circ}$ tritt Braunfärbung ein, von 75 bis $125^{\circ}$ sintert der Körper zusammen unter teilweisem Schmelzen und Verkohlen.

0,1656 gr Subst. gaben $0,1488 \mathrm{gr} \mathrm{AgBr}$

0,2540 gr Subst. gaben $0,2325 \mathrm{gr}$,

$$
\begin{aligned}
\mathrm{C}_{34} \mathrm{H}_{40} \mathrm{O}_{4} \mathrm{~N}_{2} \mathrm{Br}_{4} \text { Ber. Br } 37,16 \% \\
\text { Gef. , } 38,23 ; 38,95 \%
\end{aligned}
$$

Das einmal getrocknete Produkt, ein orangerotes Pulver, lässt sich einige Tage aufbewahren, bevor es langsam verharzt.

Lässt man die Chloroformlösung des Tetrabromides stehen, so scheiden sich allmählich weisse Krystalle in geringer Menge ab, in grösserer Menge und rascher erhält man sie, wenn man die Lösung auf dem Wasserbade erwärmt, wobei eine starke Entwicklung von Bromwasserstoff anhebt. Die neue Verbindung wird am besten aus einem Gemische von 4 Teilen Benzol und 1 Teil Aceton umkrystallisiert; weisse, glänzende, zu Büscheln vereinigte Nadeln vom Smp. 205-208 unter Zersetzung. Leicht löslich in Wasser, Methyl- und Äthylalkohol und Aceton, schwer in Äther, Chloroform, Benzol und Benzin. Unlöslich in Alkalien; an der Luft verlieren die Nadeln rasch ihren Glanz und färben sich innert wenigen Tagen braunrot.

$$
\begin{aligned}
& 0,1454 \text { gr Subst. gaben } 0,1670 \text { gr } \mathrm{AgBr} \\
& \mathrm{C}_{17} \mathrm{H}_{20} \mathrm{O}_{2} \mathrm{NBr}_{3} \text { oder } \mathrm{C}_{34} \mathrm{H}_{40} \mathrm{O}_{4} \mathrm{~N}_{2} \mathrm{Br}_{6} \text { Ber. Br } 47,05 \% \\
& \text { Gef. " } 48,87 \%
\end{aligned}
$$

Diese Analyse lässt auf ein' Tribromid schliessen; die sehr kleine Menge dieser schwer zugänglichen Substanz erlaubte keine weitere Untersuchung.

Dibromid des Oxydationsproduktes. Verdunstet man die Chloroformlösung des Tetrabromides bis zur vollständigen Entfernung des Chloroforms im Vakuum, so erhält man ein tiefrotes Harz. Man löst in Alkohol, filtriert und fällt mit Wasser ein hellgelbes Pulver aus, nach dem Trocknen löst man es in Chloroform und fällt mit Benzin ein orangerotes Pulver (ob krystallinisch, liess sich auch unter dem Mikroskop nicht sicher entscheiden). Beim Erwärmen schmilzt der 
Körper unter Zersetzung bei $123-126^{\circ}$. Schwer löslich in Benzin, sonst leicht löslich, ausser in Wasser. Erwärmen der alkoholischen Lösung bewirkt schwache Bromwasserstoffentwicklung. Unlösljch in Alkalien.

$$
\begin{array}{r}
0,2402 \mathrm{gr} \text { Subst. gaben } 0,1328 \mathrm{gr} \mathrm{AgBr} \\
\mathrm{C}_{34} \mathrm{H}_{38} \mathrm{O}_{4} \mathrm{~N}_{2} \mathrm{Br}_{2} \text { Ber. } \mathrm{Br} 22,89 \% \\
\text { Gef. , } 23,52 \%
\end{array}
$$

Auf Grund dieser Reaktionen und der Analysenzahlen war es möglich, eine Konstitutionsformel für das eigentümliche Oxydationsprodukt des Methylencampher-phenylhydroxylamines aufzustellen. Es ist für zwei Mol. dieses letzteren bei der Oxydation ein Atom Sauerstoff verbraucht worden, ferner ergab die Bestimmung des Mol.-Gewichtes eindeutig das Doppelte des ursprünglichen, also wurden zwei Molekeln Phenylhydroxylaminderivat gemeinsam oxydiert. Diese Oxydation kann nicht an der Stelle der Doppelbindung stattgefunden haben, denn die Bildung des Tetrabromides beweist das Vorhandensein von zwei Lückenbindungen, demnach erfolgte die Oxydation an den Hydroxylgruppen. Das führt zu zwei Formeln, Formel I kommt aber nicht in Betracht, da der Körper keinenfalls die Eigenschaften emes Superoxydes besitzt. Bleibt also Formel II, wonach der Körper zu einer Klasse von Verbindungen gehören würde, welche sich von der allgemeinen Formel III ableitet, so dass er als ein Disazo-dioxyd, oder auch als ein Hydrazindioxyd zu bezeichnen wäre. Dagegen wird man ihn nicht zu den Nitronen IV stellen können, denn es fehlt ihm das für Nitrone Charakteristische, nämlich die Doppelbindung zwischen einem C- und dem N-Atom.

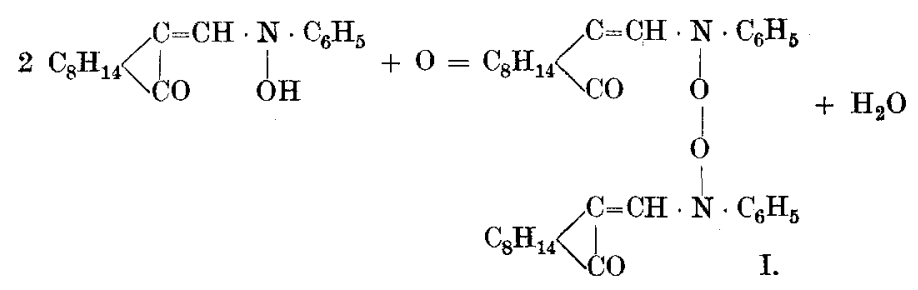

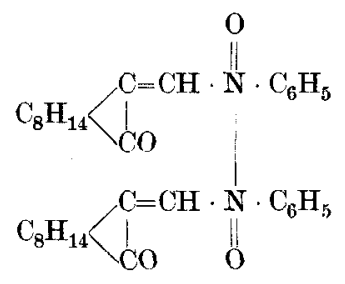

II.<smiles>[R][N+]([R1])([R1])[SH]([R])([R])=O</smiles>

III.<smiles>[R][N+]([R1])=O</smiles>

IV. 
Das Fehlen der im ursprünglichen Hydroxylaminderivate noch vorhandenen Hydroxylgruppe ist der Grund der Alkaliunlöslichkeit des Oxydationsproduktes; die ohne Bromwasserstoffentwicklung erfolgende Anlagerung von 4 Atomen Brom führt zum Tetrabromid V.

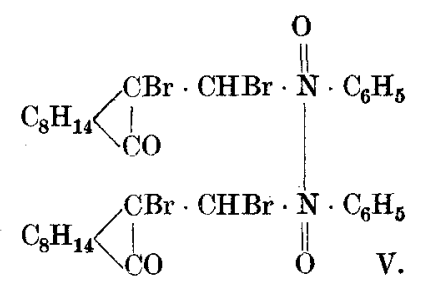

Schwieriger ist die Entstehung eines Tribromides aus der Tetrabromverbindung zu erklären; ob dieser Körper die Formel VI oder VII besitzt, wagen wir auf Grund einer Brom-Bestimmung nicht zu entscheiden. Dagegen könnte das Dibromid, das eigentliche Produkt der Bromwasserstoff-Abspaltung aus dem Tetrabromid, die Formel VIII haben, obgleich der niedrige Schmelzpunkt eher für eine Formel mit nur einem Campherrest spricht. Alle diese Bromderivate sind schwer zugänglich und sehr zersetzlich.

VI.

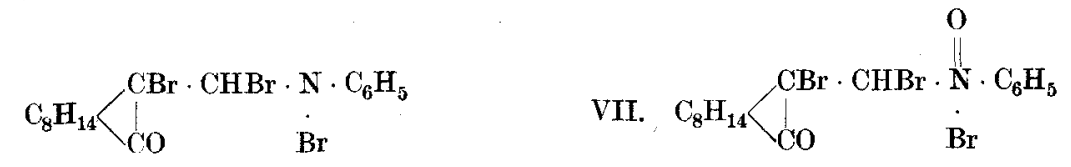

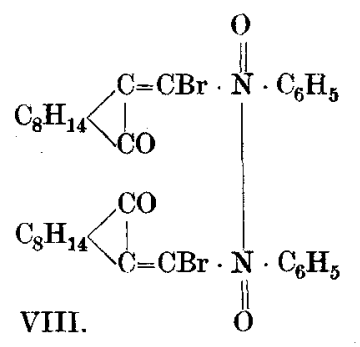

Einwirkung von Schwefelsäure auf das Oxydationsprodukt.

Das Oxydationsprodukt löst sich in Schwefelsäure von $75 \%$ langsam auf, unter Bildung einer neuen Verbindung.

2 gr reines Oxydationsprodukt ( $\mathrm{mehr}$ anzuwenden ist nicht ratsam) werden nach dem Pulverisieren in einer Achatschale in einer Stöpselflasche mit $50 \mathrm{~cm}^{3}$ Schwefelsäure von $75 \%$ übergossen und dann auf der Maschine 1/4-1/2 Stunde geschüttelt. Der Körper löst sich auf, die Lösung färbt sich dunkelgrün. Die klare Flüssigkeit wird auf Eis gegossen; nach dem Abfiltrieren des gelblich-weissen Niederschlages und gründlichem Auswaschen und Trocknen liegt ein grauweisses, 


\section{$-918$}

sandiges Pulver vor. Rohausbeute ca. 1,5 gr. Aus dem Filtrate kann durch Neutralisieren mit Soda ein weisser, schaumig ausfallender Körper von schwach basischem Charakter gewonnen werden, der sich an der Luft rasch braun färbt; er ist bis jetzt noch nicht näher untersucht worden.

Kocht man das Hauptprodukt der Reaktion mit Benzol aus, so geht ein kleiner Teil in Iuösung. Der unlösliche Rückstand wird in verdünnter Natronlauge, worin er sich leicht löst, aufgenommen; nach dem Filtrieren wird mit Salzsäure ausgefällt, der Körper lässt sich jetzt ans Alkohol unter Zusatz von Wasser gut umkrystallisieren. Nach dreimaliger Wiederholung dieses Verfahrens erhält man weisse, mikroskopisch kleine Täfelchen, die sich meist gegenseitig durchwachsen. Die Verbindung hat keinen scharfen Schmelzpunkt, sie sintert zwischen $170-175^{\circ} \mathrm{zu}$ einer dickflüssigen, braunen Masse, bei $180-185^{\circ}$ wird sie klar durchsichtig, von $190-195^{\circ}$ zersetzt sie sich unter Gasentwicklung. Sie ist in Benzol, Benzin und in Chloroform schwer löslich, beim Erwärmen mit Benzol quillt sie auf wie ein Gel. Methyl- und Äthylalkohol, Essigester, Äther und Aceton lösen leicht. Der Körper ist in Natronlauge und in Barytwasser kalt löslich, beim Erwärmen mit gelber Farbe; öäuren fällen ihn unzersetzt wieder aus. Von einer Lösung in Aceton wird Permanganat entfärbt.

$3,320 \mathrm{mgr}$ Subst. gaben $8,260 \mathrm{mgr} \mathrm{CO}_{2}$ und $2,280 \mathrm{mgr} \mathrm{H}_{2} \mathrm{O}$

3,145 mgr Subst. gaben 7,790 mgr $\mathrm{CO}_{2}$ und $2,170 \mathrm{mgr}_{2} \mathrm{O}$

5,396 mgr Subst. gaben $0,221 \mathrm{~cm}^{3} \mathrm{~N}_{2}\left(15^{0}, 724 \mathrm{~mm}\right)$

0,1738 gr Subst. gaben $6,7 \mathrm{~cm}^{3} \mathrm{~N}_{2}\left(12^{0}, 742 \mathrm{~mm}\right)$

$$
\begin{aligned}
& \mathrm{C}_{34} \mathrm{H}_{40} \mathrm{O}_{4} \mathrm{~N}_{2}+2 \mathrm{H}_{2} \mathrm{O}=\mathrm{C}_{31} \mathrm{H}_{41} \mathrm{O}_{6} \mathrm{~N}_{2} \text { Ber. C } 70,80 \quad \text { H 7,69 } \quad \text { N } 4,86 \% \\
& \text { Gef. ., 67,87; } \left.67,57,, 7,68 ; 7.72,4,54 ; 4,45 \%{ }^{1}\right\}
\end{aligned}
$$

Molekulargewichtsbestimmungen.

$$
\mathrm{C}_{34} \mathrm{H}_{40} \mathrm{O}_{4} \mathrm{~N}_{2}+2 \mathrm{H}_{2} \mathrm{O}=\mathrm{C}_{34} \mathrm{H}_{44} \mathrm{O}_{6} \mathrm{~N}_{2} \text { Mol.-Gew. ber.: } 576
$$

I. Gefrierpunktsmethode:

\begin{tabular}{|c|c|c|c|c|}
\hline Lösungsmittel & Substanz & Erniedrigung & $\begin{array}{c}\text { Mol.-Gew. } \\
\text { gef. }\end{array}$ & K \\
\hline \hline Naphtalin & & & & \\
$10,229 \mathrm{gr}$ & $0,1826 \mathrm{gr}$ & 0,143 & 879 & 6900 \\
Veratrol & & & & \\
$10.402 \mathrm{gr}$ & $0,2176 \mathrm{gr}$ & 0,295 & 352 & 6380 \\
$10,688 \mathrm{gr}$ & $0,2158 \mathrm{gr}$ & 0,427 & 301 & 6380 \\
$11.215 \mathrm{gr}$ & $0,1950 \mathrm{gr}$ & 0,370 & 299 & 6380 \\
\hline
\end{tabular}

1) Die Substanz ist sehr schwer verbrennlich, so dass die Mikroanalysen stets zu wenig Kohlenstoff ergaben. 
II. Siedepunktsmethode:

\begin{tabular}{|c|c|c|c|c|}
\hline Lösungsmittel & Substanz & Erhöhung & $\begin{array}{c}\text { Mol.-Gew. } \\
\text { gef. }\end{array}$ & $\mathrm{K}$ \\
\hline \hline Essigester & & & & \\
$12,98 \mathrm{gr}$ & $0,2614 \mathrm{gr}$ & 0,02 & 2698 & 2680 \\
$15,34 \mathrm{gr}$ & $0,2614 \mathrm{gr}$ & 0,01 & 4566 & 2680 \\
$11,96 \mathrm{gr}$ & $0,2618 \mathrm{gr}$ & 0,016 & 3666 & 2680 \\
\hline
\end{tabular}

Wie oben erwähnt, ist ein kleiner Teil des Rohproduktes in heissem Benzol löslich. Der aus dieser Lösung gewonnene Körper gleicht im ganzen Verhalten, z. B. der Alkalilöslichkeit, sehr dem in Benzol schwer löslichen Teile, der Schmelzpunkt ist sehr unscharf, aber nur wenig verschieden von dem des anderen; bei $180^{\circ}$ Braunfärbung, von 194 bis $220^{\circ}$ Sintern unter teilweiser Verkohlung.

Methyläther. 1,5 gr des reinen, mit Schwefelsäure aus dem Oxydationsprodukt erhaltenen Körpers werden in überschüssiger verdünnter Natronlauge gelöst und portionenweise mit Dimethylsulfat durchgeschüttelt, wobei die Reaktion stets alkalisch bleiben soll; die Temperatur steigt auf $40-50^{\circ}$. Allmählich scheidet sich ein gelblich-weisses Pulver ab, man verdünnt mit Wasser und wäscht nach dem Filtrieren gut aus. Der Methyläther krystallisiert aus heissem Alkohol in sehr kleinen, farblosen Prismen oder in federfahnen-artigen Gebilden; der Smp. liegt bei 226-228 . Der Körper ist leicht löslich in Äther, Chloroform, Aceton und in Benzol, schwer in kaltem Methyl- und Äthylalkohol, fast unlöslich in Bencin.

3,546 mgr Subst. gaben 9,26 mgr $\mathrm{CO}_{2}$ und 2,53 $\mathrm{mgr} \mathrm{H}_{2} \mathrm{O}$

3,606 mgr Subst. gaben 9,37 $\mathrm{mgr} \mathrm{CO}_{2}$ und 2,51 mgr $\mathrm{H}_{8} \mathrm{O}$

$3,356 \mathrm{mgr}$ Subst. gaben 8,76 mgr $\mathrm{CO}_{2}$ und 2,42 $\mathrm{mgr}_{2} \mathrm{O}$

3,386 mgr Subst. gaben 8,775 mgr $\mathrm{CO}_{2}$ und 2,48 $\mathrm{mgr} \mathrm{H}_{2} \mathrm{O}$

$4,274 \mathrm{mgr}$ Subst. gaben $0,191 \mathrm{~cm}^{3} \mathrm{~N}_{2}\left(15^{0}, 724 \mathrm{~mm}\right)$

$0,1120 \mathrm{gr}$ Subst. gaben $5,00 \mathrm{~cm}^{3} \mathrm{~N}_{2}\left(17^{0}, 742 \mathrm{~mm}\right)$

0,1422 gr Subst. gaben $6,30 \mathrm{~cm}^{3} \mathrm{~N}_{2}\left(18,5^{\circ}, 742 \mathrm{~mm}\right)$

$\mathrm{C}_{38} \mathrm{H}_{52} \mathrm{O}_{8} \mathrm{~N}_{2}$

Ber. C 72,11 H 8,28 N 4,43\%

Gef. , 71,$24 ; 70,89 ; 71,21 ; 70,70,7,98 ; 7,79 ; 8,07 ; 8,19,4,95 ; 5,03 ; 4,95 \%$

Was nun die Konstitution dieses neuen, mit Schwefelsäure entstandenen Körpers betrifft, so lässt sie sich in Anbetracht des schwer zugänglichen und schwierig in analysenreinem Zustande zu erhaltenden Materiales heute noch nicht vollkommen aufklären. So viel aber geht aus unseren Untersuchungen mit Sicherheit hervor, besonders aus den 
Analysen des ganz rein dargestellten Methyläthers, dass pro Molekel Oxydationsprodukt 2 Mol. Wasser aufgenommen worden sind. Zieht man ferner in Betracht, dass die Substanz leicht in Alkalien und in Barytwasser löslich ist, sich also ganz ähnlich verhält, ja sogar noch sauerer ist, als das ursprüngliche Methylencampher-phenylhydroxylaminderivat, welches in Barytwasser nicht löslich ist, so muss man zu der Ansicht gelangen, dass eine Hydroxylgruppe am Stickstoff sich befindet. Dies führt zu der Formel IX, durch welche auch die Unbeständigkeit gegenüber Permanganat erklärt würde. Die damit tautomere Formel X, die natürlich auch in Betracht gezogen werden muss, scheint uns viel weniger wahrscheinlich, weil dadurch der stark sauere Charakter der Substanz nicht genügend erklärt würde. Manasse ${ }^{1}$ ) hat gezeigt, dass der, von ihm dargestellte Oxycampher XI eine derartig schwache Säure ist, dass seine Salze durch Wasser vollkommen hydrolysiert werden. Vielleicht dürfen wir annehmen, dass die zweite Form $(\mathrm{X})$ unter dem Einflusse des Alkalis in die erste (IX) übergeht. Unsere Bestimmungen des Molekulargewichtes geben leider kein eindeutiges Resultat; auf Grund einiger Messungen in Veratrol als Lösungsmittel könnte der Körper sogar die einfache Formel (halbe Formel) $\mathrm{C}_{17} \mathrm{H}_{22} \mathrm{O}_{3} \mathrm{~N}$ haben.

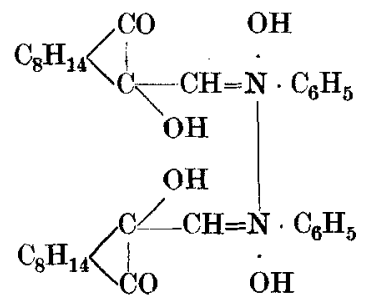

IX.

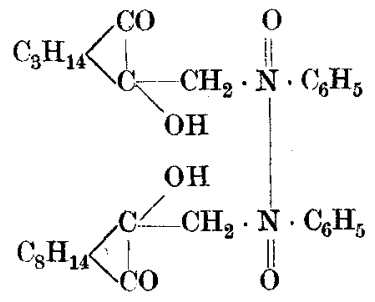

$\mathrm{X}$.

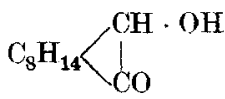

XI.

Wenngleich auch die von uns aufgestellte Konstitutionsformel des Oxydationsproduktes einen hohen Grad von Wahrscheinlichkeit besitzen dürfte, so betrachten wir sie doch als noch nicht ganz sicher (es ist uns z. B. noch nicht gelungen, ein wohl definiertes Reduktionsprodukt dieses Körpers zu erhalten), und beträchtlich unsicher ist noch die Formel des durch Wasseranlagerung entstandenen Produktes. Trotzdem glauben wir aber in dem Oxydationsprodukte des Methylencampher-phenylhydroxylamines einen bemerkenswerten Vertreter einer

1) Manasse, B. 35, 3811 (1902). 
neuen Klasse von Verbindungen gefunden zu haben. Wir hoffen zuversichtlich, dass es uns bei Gelegenheit unserer Arbeiten über die Kondensation von Phenylhydroxylamin mit Enolen u. dgl. gelingen wird, noch einmal zu einem solchen Körper zu gelangen.

Schliesslich war es noch nötig, zu zeigen, dass bei der Kondensation von Oxymethylencampher mit Phenylhydroxylamin tatsächlich ein Körper entsteht, der die von uns angenommene Konstitution besitzt, und dass nicht etwa eine Umlagerung zu einem A min o ph e no la bkö m m linge des Methylencamphers sich vollzog, was wegen der sauren Eigenschaften des Körpers immerhin nicht ausgeschlossen erschien. Deshalb wurde das p-Aminophenolderivat selbst dargestellt. $5 \mathrm{gr}$ Oxymethylencampher wurden in möglichst wenig Methylalkohol mit einer Lösung von $3 \mathrm{gr}$ p-Aminophenol in 30-proz. Essigsäure vermischt. Schwache Erwärmung und Trübung, bald Abscheidung eines dickflüssigen Öles; beim Stehenlassen und Verreiben mit Wasser wird es allmählich fest. Die Reinigung machte Schwierigkeiten; man löste in kaltem Eisessig und goss in viel Wasser. Nach dem Auswaschen und Trocknen des Niederschlages erhielt man ein graugrünes Pulver, ohne bestimmten Schmelzpunkt, leicht löslich in Alkalien; aber weitere Versuche, den Körper krystallisiert zu erhalten, waren fruchtlos. Dagegen gelang leicht die Darstellung eines Methyläthers und eines Acetylesters.

Methyläther des Methylencampher-p-aminophenols.

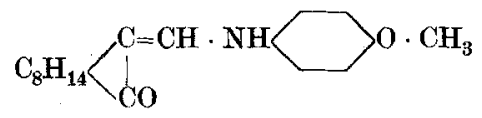

5 gr des Phenolderivates, in wenig Alkohol gelöst, wurden nach dem Versetzen mit verdünnter Natronlauge portionenweise mit 3 gr Dimethylsulfat geschüttelt. Nach dem Erkalten verdünnte man mit Wasser, saugte das abgeschiedene weisse Pulver ab und krystallisierte aus kochendem Äthylalkohol um. Feine weisse Blättchen vom Smp. $169-172^{\circ}$, leicht löslich in Chloroform, in heissem Methyl- und Äthylalkohol, schwer löslich in kaltem Äther, Ligroin und Benzol.

0,3118 gr Subst. gaben $13,4 \mathrm{~cm}^{3} \mathrm{~N}_{2}\left(16^{\circ}, 749 \mathrm{~mm}\right)$

$$
\begin{array}{r}
\mathrm{C}_{18} \mathrm{H}_{23} \mathrm{O}_{2} \mathrm{~N} \text { Ber. } \mathrm{N} \mathrm{4,91 \%} \\
\text { Gef. , } 4,91 \%
\end{array}
$$


Acetylderivat des Methylencampher-p-aminophenols.<smiles>COC(=O)OCCCNC=C1C(=O)C1c1ccccc1</smiles>

6,5 gr des p-Aminophenolderivates wurden, mit der doppelten Menge Essigsäure-anhydrid übergossen, drei Stunden auf dem Wasserbade erwärmt, wobei eine rotbraune Lösung entstand. Als dann das überschüssige Essigsäure-anhydrid im Vakuum bei einer Temperatur von $50-60^{\circ}$ abdestilliert wurde, hinterblieb ein krystallinischer Rückstand; er wurde mit wenig Äther gewaschen und aus heissem Äthylalkohol umkrystallisiert. Das Acetylderivat bildet weisse Blättchen vom Smp. $221-223^{0}$ :

$$
\begin{gathered}
0,1704 \text { gr. Subst. gaben } 6,5 \mathrm{~cm}^{3} \mathrm{~N}_{2}\left(15^{0}, 749 \mathrm{~mm}\right) \\
\mathrm{C}_{19} \mathrm{H}_{23} \mathrm{O}_{3} \mathrm{~N} \text { Ber. } \mathrm{N} 4,47 \% \\
\text { Gef. , } 4,38 \%
\end{gathered}
$$

Leicht löslich in kaltem Chloroform, Aceton, Benzol, heissem Methyl- und Äthylalkohol, schwer in kaltem Äther und in Ligroin. Die Eigenschaften des p-Aminophenolderivates, seines Methyläthers und Acetylderivates sind so grundverschieden von denen des Phenylhydroxylaminderivates und seiner Abkömmlinge, dass eine AminophenolUmlagerung bei der Kondensation von Oxymethylencampher mit Phenylhydroxylamin ausgeschlossen ist.

Basel, Anstalt für Organische Chemie. 